\title{
SMFF Computation for Probe of Online Magnetic Flux Leakage Detection System with combination of ANSYS and Virtual Work Method
}

\author{
Jin-zhong $\mathrm{CHEN}^{1, a}$, Yi-lai Ma ${ }^{1, b^{*}}$, Ren-yang $\mathrm{HE}^{1, \mathrm{c}}$ \\ ${ }^{1}$ Pressure Pipeline Division, China Special Equipment Inspection and Research Institute, \\ Beijing, China \\ acchenbeiter@aliyun.com, ${ }^{\mathrm{b}}$ chantal1314@163.com, ${ }^{\mathrm{C}}$ herenyang@163.com
}

Keywords: ANSYS, Virtual work method, Static magnetic field force (SMFF), Nonlinear.

\begin{abstract}
Numerical analysis of static magnetic field force for probe of online magnetic flux leakage detection system was conducted by combining ANSYS and Virtual work method. The result shows that combination of ANSYS and virtual work method could accurately calculate the static magnetic field force between the probe and the drill pipe. In addition, numerical solution in 3D model could reduce the calculation error caused by nonlinear distribution of magnetic induction $\mathrm{B}$ between the probe and the cylindrical surface of drill pipe. What's more, the graphs of static magnetic field force were obtained by the different thickness of the yoke and air gap thickness between the probe and the drill pipe. The graphs show that the static magnetic field force changed linearly with yoke thickness. However, it changed nonlinearly with the air gap thickness between the probe and the drill pipe. It can be concluded that the effect for static magnetic field force of latter was greater than that for static magnetic field force of former.
\end{abstract}

\section{Introduction}

Drill pipe online magnetic flux leakage detection system can automatically detect the drill pipe in the progress of drilling [1]. In the testing progress, the six permanent probes in uniform arrangement which absorb on the drill pipe with the magnetic attraction will excite the drill pipe and detect the defect of drill pipe online. The quantitative analysis of static magnetic field force has great significance due to static magnetic field force (SMFF) between the probes and drill pipe will directly affect their tightness.

This paper created a 3D model of drill pipe and probe with ANSYS and analyzed quantitatively the SMFF between the probes and drill pipe with combination of ANSYS and virtual work method. Also the effects about different air gap thickness between the probe and the drill pipe and different yoke thickness to SMFF have been down quantitatively.

\section{Virtual Work Method Principle}

Virtual work method is based on principle of conservation of energy and principle of virtual displacement. In the condition of constant current and constant magnetic flux, when a part of the electromagnetic device has a small displacement (I.e. can be true displacement and a virtual displacement) in some direction, the magnetic energy of whole system will change with the displacement of the part and the part will endure the magnetic force in the progress of moving. In the condition of constant current, the size of magnetic force equal to the incremental magnetic energy due to the incremental unit displacement of one part [2]. In the condition of constant magnetic flux, the size of magnetic force equal to the incremental magnetic resonance energy due to the incremental unit displacement of one part. In the method of FEA, magnetic vector potential method is facility to calculate the magnetic force with the condition of constant current and the scalar magnetic potential method is facility to calculate the magnetic force with the condition of constant magnetic flux. In this paper, the calculation of SMFF between the probe and drill pipe adapt the scalar magnetic potential 
method because there is no current between the probe and drill pipe. So the calculation formula of magnetic force is derived on base of constant magnetic flux.

\section{Mathematical Model}

According to the virtual work method, when the ferro magnetic material has a small displacement in the q direction, the SMFF of ferro magnetic material in the same direction is calculated as follows:

$$
F_{q}=(-\partial W) / \partial q
$$

where, the $F_{q}$ is the magnetic force in $q$ direction and the $W$ is the total magnetic energy.

The total magnetic energy $W$ is predicted by the following Eq. (2).

$$
W=\int_{V}\left[\int_{0}^{B} H \cdot d B\right] d V
$$

where, the $V$ is the computational domain and the $B$ is the magnetic induction and the $H$ is magnetic field intensity.

In the progress of FEA [3], computational domain $\mathrm{V}$ will be discretized by a series of hexahedral elements when the grid is divided by the hexahedral element and the $\mathrm{W}$ is the total magnetic energy of all magnetic energy of hexahedral unit. So Eq. (2) can be discretized in Eq. (3) [4].

$$
W=\sum_{e=1}^{N}\left[\int_{0}^{B} H_{e} \cdot d B_{e}\right] d V_{e}
$$

where, $N$ is number of total hexahedral elements and $V_{e}$ is the volume of hexahedron element.

When the computational domain is discretized by the first-order hexahedron element, integration of Eq. (3) gives the following analytical expression:

$$
W=\sum_{e=1}^{N} \frac{B^{2}}{2 \mu_{e}} V_{e}
$$

where, the $\mu_{e}$ is the magnetic conductivity of the first-order hexahedron element.

Inserting Eq. (4) into Eq. (1), we can get Eq. (5) that is the final discrete calculation formula of magnetic force by scalar magnetic potential method. Eq. (5) consist of $B$ and $r_{e}$.

$$
F_{q}=-\sum_{e=1}^{N}\left[\frac{V_{e}}{2} \frac{\partial B^{2}}{\partial q} r_{e}+\frac{V_{e}}{2} B^{2} \frac{r_{e}}{\partial B^{2}} \frac{\partial B^{2}}{\partial q}+\frac{\partial V_{e}}{\partial q} \frac{B^{2}}{2} r_{e}\right]
$$

where, the $r_{e}$ is the node reluctivity.

The magnetic induction $B$ can be calculated by Maxwell equations. The simplified Maxwell equations show in Eq. (6) according to the condition of static magnetic field.

$$
\left\{\begin{array}{l}
\nabla \times H=0 \\
\nabla \cdot B=0
\end{array}\right.
$$

where, the $\nabla$ represents the Hamiltonian operator.

\section{Finite Element Model and Material properties}

A 3D FEA model of drill pipe and probes were created shown in Fig. 1, with the 400mm long and $12.7 \mathrm{~mm}$ thick drill pipe. The diameter of drill pipe is $127 \mathrm{~mm}$ shown in Fig. 1. Two pieces of opposite polarity of permanent magnets have the same size shown in Fig. 1, with 70mm length, 60mm width and $70 \mathrm{~mm}$ height. The exciting yoke is used to form the magnetic circuit with $200 \mathrm{~mm}$ length, $60 \mathrm{~mm}$ width and $20 \mathrm{~mm}$ height. The smallest gap between the drill pipe and the permanent magnet are $6 \mathrm{~mm}$. 
The attributes of air, magnet, magnetic yoke and drill pipe material should be defined in magnetic flux leakage model [5-6].

(1) Air Its magnetic characteristic is linear. Define its relative magnetic conductance as 1.

(2) Drill pipe Its material characteristic should be determined according to chosen pipes. Commonly used drill pole materials are Q235, X52, X60, X70 and some other various brands. Commonly used X52 steel is chosen in experiment model.

(3) Magnetic yoke Low carbon steel with high magnetic conductance is chosen. St37 is chosen.

(4) Magnet Permanent magnet is described through magnetic coercive force vector and demagnetization B-H curve [7-8]. Not only magnetic characteristic curve but also its magnetizing direction should be given. Permanent magnetic material Nd-Fe-B magnet, whose residual magnetism, coercive force and static biggest energy product have an optimal solution, is chosen as excitation device in most magnet leakage testing. Nd-Fe-B permanent magnet N52 is chosen in this experiment.

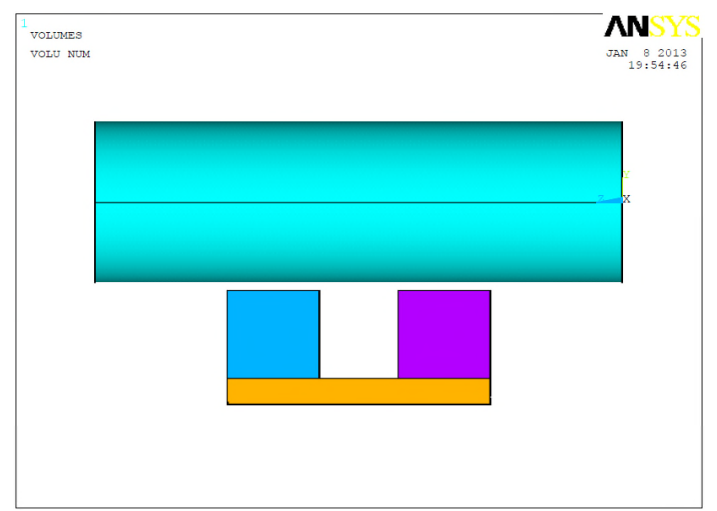

1-Drill pipe; 2-Magnet; 3-Magnet; 4-Magnetic yoke

Fig. $13 \mathrm{D}$ finite element model of drill pipe and probe.

ANSYS apply the natural boundary condition on interface of the parts and apply Neumann boundary condition on all external face in the analysis of static magnetic field [9-10]. The natural boundary condition is to define that the tangential component of $\mathrm{H}$ and the normal component of $\mathrm{B}$ is continuous on the interface of parts. Also, the Neumann boundary condition is to define the $\mathrm{H}$ is tangent to the surface boundary and the normal component of $\mathrm{H}$ is zero on the surface boundary. In addition to the two kinds of boundary conditions, this paper still applies the virtual displacement boundary condition on the cylindrical surface of drill pipe.

\section{Numerical Solution and Analysis}

Fig. 2 and Fig. 3 show 3D front vectors elevation and right vectors elevation of the magnetic induction B solved by Eq. (6) respectively. The results show that the distribution of magnetic induction $\mathrm{B}$ on air gap is nonlinear and the magnetic induction B is uniform which is coincident with the theory.
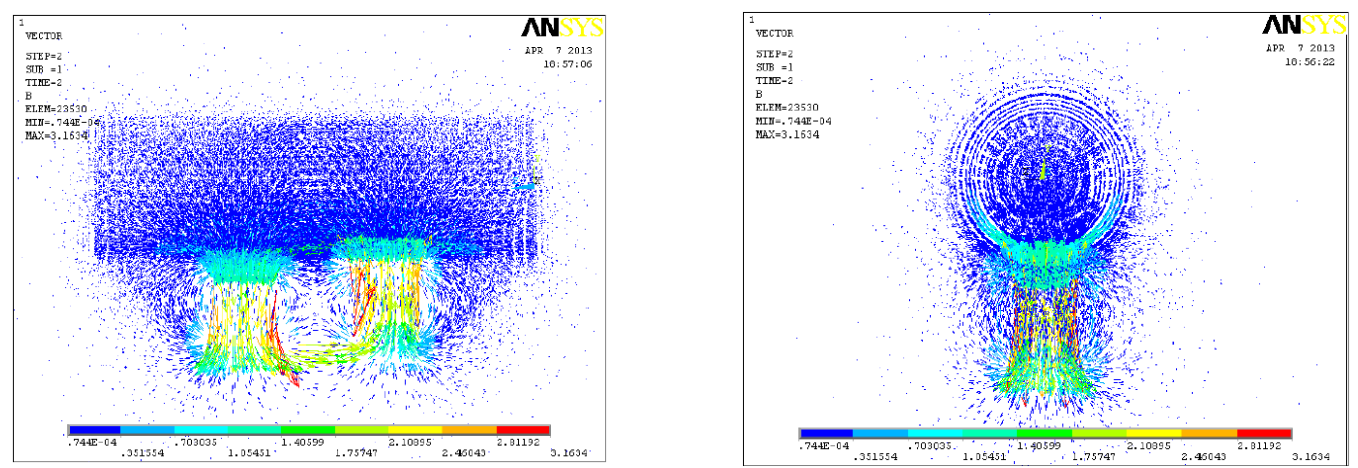

Fig. 2 3D Vector of Magnetic induction B. Fig. 3 3D Vector of magnetic field strength $H$. 
The SMFF of all nodes in the model are shown in the Fig. 4 and Fig. 5. Due to the existence of cylindrical surface of drill pipe, we can get the Y component of SMFF of node on middle section of model is maximum from the Fig. 4 and Fig. 5. So the number of node has been obtained and shown in the Fig. 6. From the Fig. 6, we can get the number of node is symmetrical about the symmetry plane of model. This is due to the symmetrical characteristic of the calculated model which also bring about the $\mathrm{X}$ component and $\mathrm{Z}$ component of SMFF of node is zero.

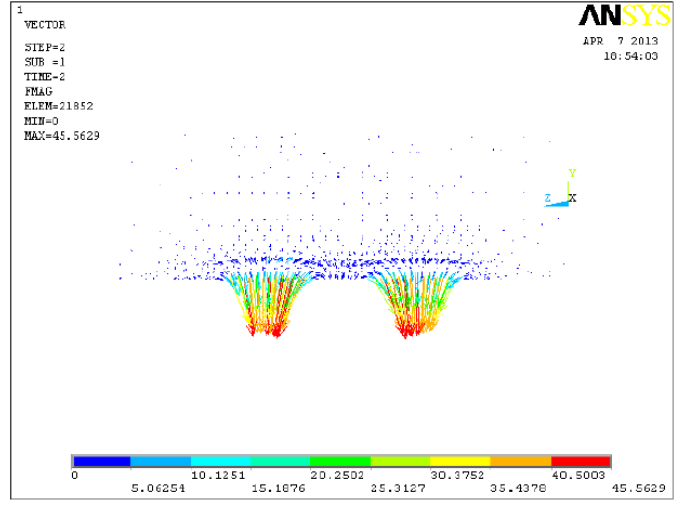

Fig. 4 3D front vectors elevation of SMFF.

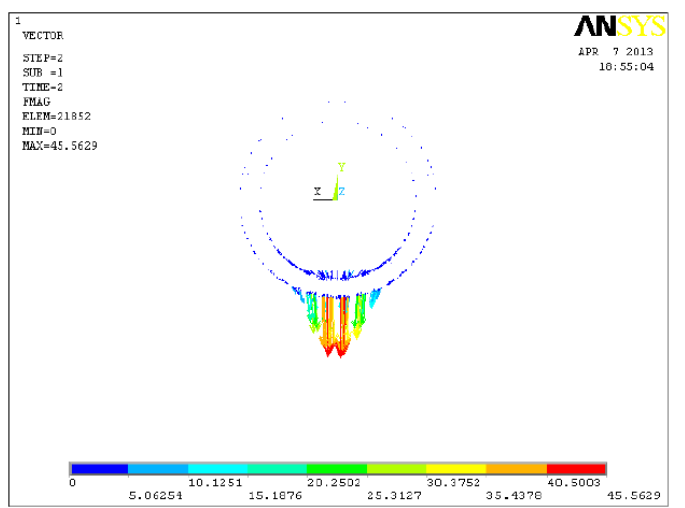

Fig. 5 3D right vectors elevation of SMFF.

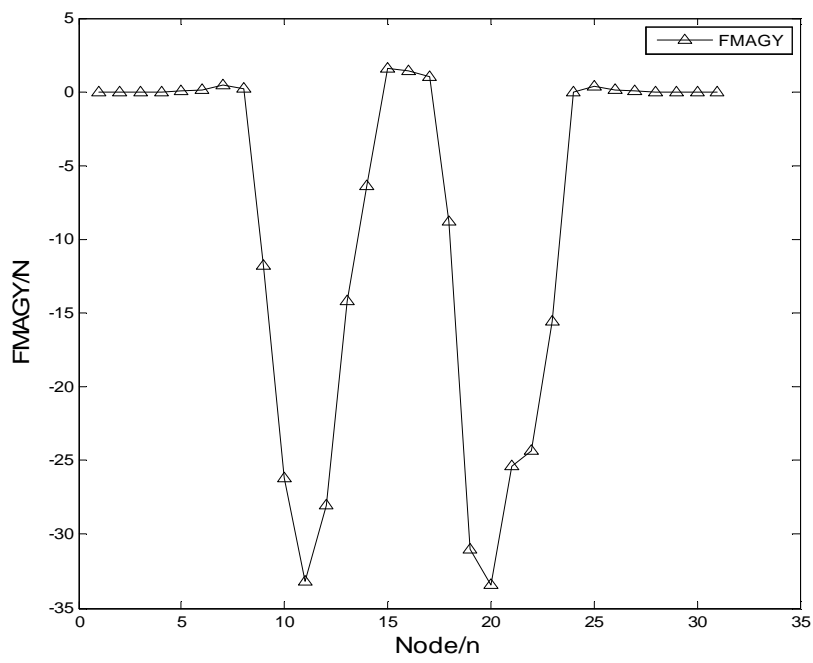

Fig. 6 Y component of SMFF of node on middle section.

The Table 1 shows the total SMFF between the drill pipe and probe. The table shows that the SMFF between the drill pipe and probe mainly appears in the $\mathrm{Y}$ component and the $\mathrm{X}$ component and $\mathrm{Z}$ component is almost zero and the $\mathrm{Y}$ component of SMFF is up to $3478 \mathrm{~N}$.

Table 1. Static magnetic field force between the probe and the drill pipe.

\begin{tabular}{ccc}
\hline Force-X/N & Force-Y/N & Force-Z/N \\
\hline 0.65 & 3478 & 0.62 \\
\hline
\end{tabular}

Because the different thickness of air gap and yoke will bring about the different SMFF, a serious different SMFF shown in Fig. 7 has been calculated by changing the thickness of air gap. We can get that the SMFF decreases in the way of nonlinear with the increasing of the air gap thickness from Fig. 7. This phenomenon tells the effect of changing thickness of air gap decreases gradually on reduction of SMFF. 

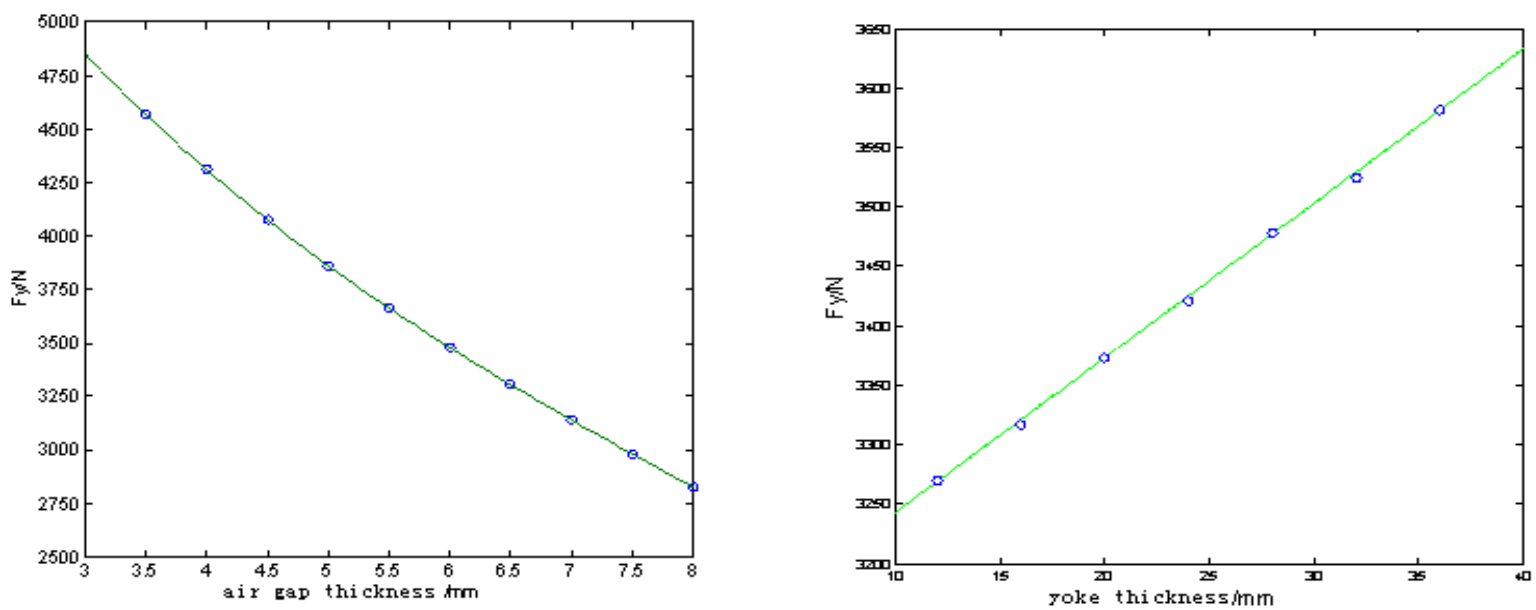

Fig. 7 Effect of different air gap thickness on static Fig. 8 Effect of different yoke thickness on static magnetic field force.

magnetic field force.

Because the different thickness of air gap and yoke will bring about the different SMFF, also a serious different SMFF shown in Fig. 8 has been calculated by changing the thickness of yoke. We can get that the SMFF decreases in the way of linear with the increasing of the yoke thickness from the Fig. 8. This phenomenon is because of the linear changeable thickness of yoke make the reluctivity of field changing linearly which ultimately lead to SMFF changing in the way of linear.

Compare Fig. 7 with Fig. 8, we can tell effect of thickness of air gap is bigger than the effect of thickness of yoke which is mainly because of the reluctivity of air gap is much more than the reluctivity of yoke.

\section{Conclusions}

(1) The SMFF of 3d model has been calculated precisely by the combination of ANSYS and virtual work method which decreases the error caused by the nonlinear distribution of magnetic induction intensity B which is caused by the existence of cylinder surface of drill pipe.

(2) The effect of thickness of air gap and thickness of yoke on the SMFF has been achieved respectively. The calculation shows that the changeable thickness of air gap brings about nonlinear change of SMFF. On the contrary the changeable thickness of yoke brings about linear change of SMFF. What's more, the effect of thickness of air gap on the SMFF is bigger than the effect of thickness of yoke on the SMFF. So we can optimize structure parameters of system by change the thickness of air gap and yoke.

\section{References}

[1] Y. L. Ma, R. Y. He, J. Z. Chen, A Method for Improving SNR of Drill Pipe Leakage Flux Testing Signals by Means of Magnetic Concentrating Effect, IEEE T. Magnetics, 51(9) (2015) 489-492.

[2] M. Andriollo, G. Martinelli, A. Morini, et al. FEM calculation of the LSM propulsion force in EMS_MAGLEV trains, IEEE T. Magnetics 32(5) (1996) 5064-5066.

[3] A. J. Deeks, J. P. Wolf, A virtual work derivation of the scaled boundary finite-element method for elastostatics computational mechanics, Comput. Mech. 28(6) (2002) 489-504.

[4] R. Sanchez-Grandia, R. Vives-Fos, V. Aucejo-Galindo, Magnetostatic Maxwell's tensors in magnetic media applying virtual works method from either energy or co-energy, Eur. Phys. J. Appl. Phys. 35(1) (2006) 61-68.

[5] E. Norouzi, H. Ravanbod, Optimisation of the flux distribution in magnetic flux leakage testing, Insight, 51(10) (2009) 563-567. 
[6] X. C. Yuan, C. L. Wang, F. Z. Ji, X. Z. Zuo, A magnetic flux leakage analysis model based on finite element neural network, Insight, 53(9) (2011) 482-486.

[7] Y. Li, J. Wilson G Y Tian, Experiment and simulation study of 3D magnetic field sensing for magnetic flux leakage defect characterisation, NDT\&E Int. 40 (2007) 179-184.

[8] F. I. Al-Naemi, J. P. Hall, A. J. Moses, FEM modelling techniques of magnetic flux leakage-type NDT for ferromagnetic plate inspections, J. Magn. Magn. Mater. 304 (2006) 790-793.

[9] W. Cui, S. L. Huang, W. Zhao, J. F. Burd, The effect of the defect location on the finite element modelling of defect MFL fields, Insight, 48(7) (2006) 402-405.

[10] Y. Tang, M. C. Pan, F. L. Luo, X. L. Tan, Experimental and finite element analysis study of 3D magnetic field sensing for pulsed magnetic flux leakage defect characterisation, Insight, 53(9) (2011) 497. 